\title{
MODELING OF RADIATION-INDUCED ELECTRIC CURRENT IN THE MATERIALS OF FINELY DISPERSED STRUCTURE
}

\author{
M.E. ZHUKOVSKIY ${ }^{1 *}$, M.B. MARKOV, R.V. USKOV ${ }^{1}$ AND L.V. KUZNETSOVA ${ }^{2}$ \\ ${ }^{1}$ Keldysh Institute of Applied Mathematics of RAS (KIAM), Moscow, Russia \\ ${ }^{2} 12^{\text {th }}$ Central research Institute of the Ministry of defense of the Russian Federation, Moscow, Russia \\ * Corresponding author. E-mail: usermath@mail.ru
}

DOI: 10.20948/mathmontis-2020-47-6

Summary. The technique of Monte Carlo modeling of radiation-induced electric currents in heterogeneous finely dispersed medium with direct consideration of their microstructure is worked out. The main attention is paid to developing the method of the construction of a geometric model of the polydisperse structures. The method based on random tracing algorithm is intended for implementation on heterogeneous computing clusters with using the graphical processors and the CUDA parallelization of calculations. The geometric model includes a detecting system for statistical evaluation of the desired physical quantities (electric current density). A computational experiment was performed to study the basic regularities of generation of electrical currents arising in polydispersed mfterial being under X-radiation. The results of the experiment showed the irradiation of the object under study produces electric currents with a sharply inhomogeneous spatial structure. Inhomogeneities occur near the boundary surfaces between the binder and inclusions.

\section{INTRODUCTION}

Heterogeneous materials of finely dispersed structures are widely used in mechanical engineering, heat power engineering, rocket, aviation, chemical and other industries. This is because these materials provide the required strength, thermal, hydraulic, technological properties and can operate at high temperatures and pressures. Such materials are used, for instance, in protection of structures from intensive energy flows [1], creation of solid propellants [2,3].

Investigation of radiation-induced electrical effects in heterogeneous finely dispersed media is very actual for researching the protective and functional properties of such media being under radiation [4-9]. Heterogeneous dispersed structures are the materials having huge number of inner boundaries between homogeneous components. The presence of these boundaries leads to generation of electrical phenomenon due to the lack of electronic equilibrium near them [10-12].

Mathematical modeling of radiation-induced electrical effects in finely dispersed media involves the development of radiation transport simulation algorithms as well as the construction of a geometric model of a substance with a direct resolution of its microstructure.

The transport of radiation in heterogeneous materials of complex geometric structure is cascade process and characterized by the fact that the particle pathways are comparable with the size of the inhomogeneities of the medium. In this situation, a detailed simulation of each collision of radiation particles with atoms of the medium is required [13]. Statistical

2010 Mathematics Subject Classification: 97M50, 97N50, 93A30.

Key words and Phrases: Finely dispersed medium, Radiation induced current, Monte Carlo simulation. 
algorithms of radiation cascade transport modelling are worked out considering the features of radiation transport in finely dispersed media $[14,15]$.

The main attention in this work is paid to the construction of a geometrical model of a dispersed structure. The model includes the detector (registration) system for statistical estimation of the electrical current in an irradiated object.

Various algorithms can be used to construct a geometric model of the material (placement of microstructure particles with specified geometric properties inside the sample). The most popular of these is the algorithm of Lubachevsky-Stillinger [16-18]. The algorithm simulates the process of mechanical compression of a set of solid particles. There are other techniques for construction of geometrical model of materials in question [19, 20].

These algorithms are poorly parallelized on GPUs due to their complex internal logic and therefore cannot be integrated into common code designed for heterogeneous computing clusters (HCC).

A method of creating the geometrical model of the irradiated object based on ray-tracing algorithm [21] is worked out in this work. The method has almost unlimited scalability and is easily implemented on the graphics subsystem of the HCC.

The developed code for supercomputer simulation of radiation-induced electric currents in heterogeneous dispersed materials with direct consideration of their microstructure is implemented on heterogeneous computing clusters.

The results of a computational experiment to calculate the current density in a fragment of finely dispersed material show that electric currents with a sharply inhomogeneous spatial structure are formed during irradiation of the object under study. Inhomogeneities occur near the boundary surfaces between the binder and inclusions.

\section{GEOMETRICAL MODEL OF THE FINELY DISPERSED STRUCTURES}

Base characteristics of the dispersed medium are the size of suspended particles in dispersed systems and dispersity (relative volume fraction of suspended particles of every type). It is assumed in this paper that all particles of given type are of the same size.

The geometric model also includes a model of the detector system for the statistical evaluation of the required physical quantities (electrical current). The detector system intended for the statistical estimation of functionals on the space of solutions of the transport equation includes a set of "detectors", spheres of a specified size and location within which the events of the interaction of the radiation quanta and the secondary particles with the material are recorded.

The detectors must be isolated from each other (should not intersect) and the entire volume of the detector should be inside the given matter (in the context of the considered media, they should not "capture" the boundaries between homogeneous components).

It can consider the model of the dispersed matter with the detectors as a polydisperse medium consisting of some types of solid nonoverlapping objects (inclusions): suspended particles and detectors. However, several detectors may be inside a single particle.

Let some object be a polydisperse medium consisting of a binder and $N$ types of suspended spherical particles of radius $r_{n}(n=1, \ldots, N)$.

The developed algorithm for creating a geometric model has the following structure.

Initially, the placement of inclusions of the $1^{\text {st }}$ type is constructed.

1. The coordinates $\left(X_{\min }, Y_{\min }, Z_{\min }\right)$ and $\left(X_{\max }, Y_{\max }, Z_{\max }\right)$ of the object are determined; 
2. $M_{x}^{n=1}=\left(Z_{\max }-Z_{\text {min }}\right) \cdot\left(Y_{\max }-Y_{\min }\right) / \pi r_{n=1}^{2}$ beams are drawn from a random point on the plane $x=X_{\text {min }}$ along the $X$-axis; $M_{y}^{n=1}=\left(Z_{\max }-Z_{\min }\right) \cdot\left(X_{\max }-X_{\min }\right) / \pi r_{n=1}^{2}$ beams are drawn from a random point on the plane $y=Y_{\min }$ along the $Y$-axis and $M_{z}^{n=1}=\left(Y_{\max }-Y_{\min }\right) \cdot\left(X_{\max }-X_{\min }\right) / \pi r_{n=1}^{2}$ beams are drawn from a random point on the plane $z=Z_{\min }$ along the $Z$-axis;

3. The intersection points of the beams with boundary surfaces of homogeneous parts of the object are calculated (fig. 1).

4. A random point (center of a particle of $1^{\text {st }}$ type) on every interval between two consecutive intersection points (segments 1-2, 3-4 and 5-6 in fig. 1) is played.

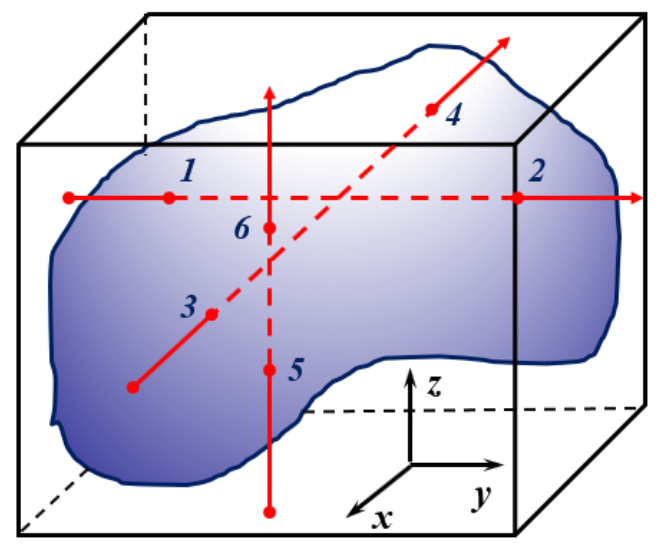

Fig. 1. Scheme of particle placement algorithm

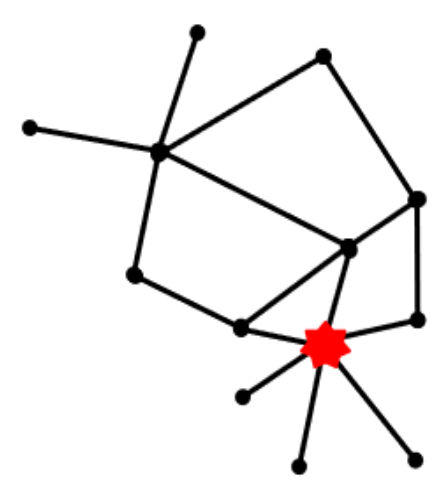

Fig. 2. Scheme of particle excluding

The next stage of the algorithm is to filter (exclude) particles according to the following criteria.

- The particles should not intersect the boundary surfaces of the homogeneous parts of the object (if intersection takes place, particle is excluded from corresponding set);

- The particles must be isolated from each other.

Elimination of mutual intersections of detectors is carried out by using the following method.

- It is built a graph on the set of constructed points (centers of the inclusions). The nodes of the graph are the centers of the particles. The edges of the graph are constructed between two nodes for which the distance between the centers of the particles is less than $2 r_{n=1}+S_{n=1}\left(S_{n=1}\right.$ is special value that restricts the minimum distance between the particles);

- The node of the graph having the maximum number of edges is defined. It is excluded from the graph (fig. 2).

Last step is repeated until there are no edges left in the graph.

The algorithm is repeated with additional beams if a number of inclusions is less then requiered.

The set of detectors is constructed after the geometrical model is built with inclusions of all types. The developed algorithm is used for the construction of the detector set but there is one 
exception. Some of the detectors may be located entirely inside the inclusions to estimate the desired value in suspended particles.

A fragment of geometrical model consisting of epoxy binder, metal or dielectric inclusions (blue spheres) and a set of detectors (magenta spheres) is shown in fig. 3 (the image is enlarged for clarity).

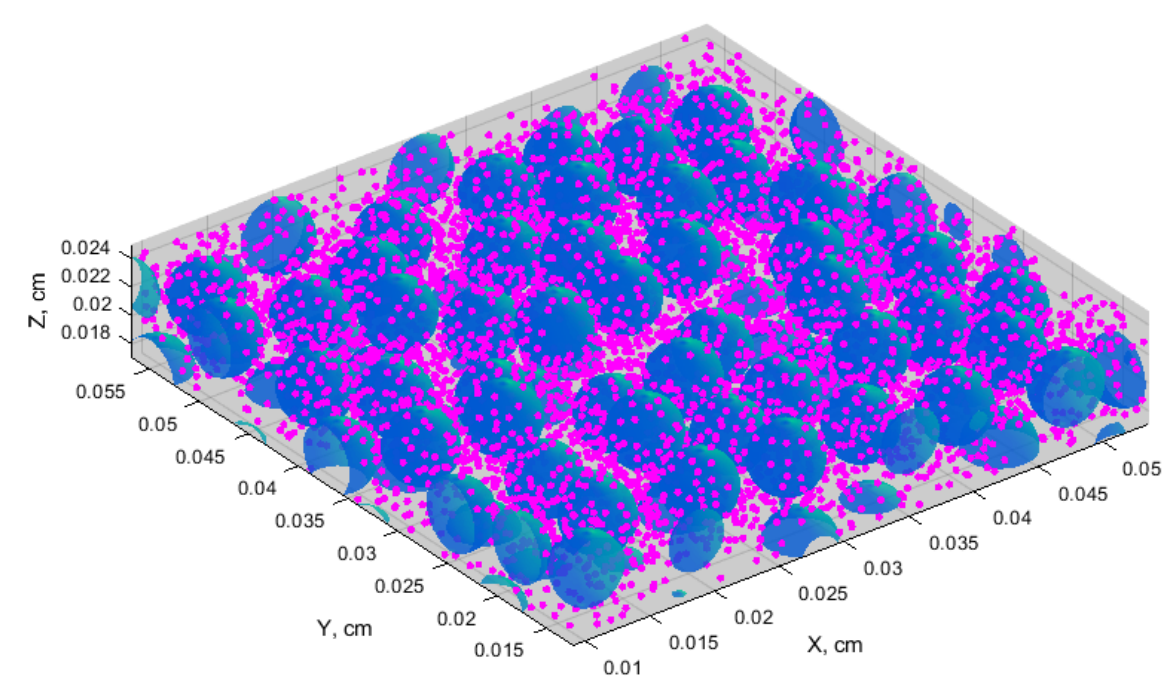

Fig. 3. A fragment of geometrical model constructed by use of developed algorithm

All inclusions are closed, but appear cropped due to image magnification.

\section{MODELING OF THE RADIATION TRANSPORT}

The complicated process of particle transport through the matter can be represented by a sequence of elementary processes of the interaction between the particle and the atoms of matter (particle trajectory). These processes include the scattering, braking or disappearance of the particle due to absorption or escape from the considered system (from the object). This representation is convenient for modelling the radiation transport by the Monte-Carlo method.

The transport of the particles accompanied by the birth of secondary particles in cascade processes of the interaction of the radiation with matter is described by a system of integral equations.

$$
Q=Q_{1}+\int k\left(x^{\prime}, x\right) Q\left(x^{\prime}\right) d x^{\prime}=Q_{1}+K Q .
$$

Here $x=(\mathrm{r}, \Omega, E)$, where $\mathrm{r}, \Omega, E$ are coordinates, direction of motion and energy, respectively; $Q(x)$ is the density of collisions and $Q_{1}(x)$ is the density of the first collisions; $k=\left(x, x^{\prime}\right)$ is the kernel of the integral operator and has the meaning of probability density of $x^{\prime} \rightarrow x$ transition. 
Equation (1) is true for every type of particles of cascade. The previous generation particle flux is the next generation particle source.

The objective of the radiation transport theory is to compute the readings of detector located in the field of radiation. The desired (measured) values are presented as the readings of some detector and are written as functional on the space of the transport equation solutions. We consider such registering facilities (detectors) whose readings are equal to the sum of the contributions of some particle's collisions in a sensitive volume of the detector (additive detectors). To evaluate the desired measured value by the Monte Carlo method, the random trajectories of the particles are simulated (fig. 4).

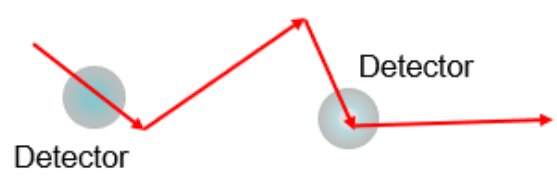

Fig. 4. Particle trajectory

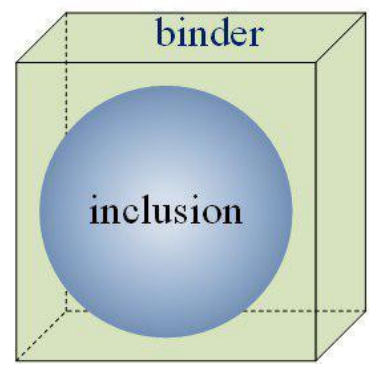

Fig. 5 A fragment of dispersed material

The contributions of these trajectories to the detector's measurable value are summed up. The particle trajectory construction is performed according to the chosen physical model of the interaction between the radiation and matter.

Trajectories are simulated using the individual computational algorithms for each type of particle considering their physical properties [13].

The developed algorithm is described in detail in [15].

\section{RESULTS OF THE COMPUTATIONAL EXPERIMENT}

This section presents the results of computational experiments on simulation of radiationinduced electric currents in heterogeneous materials of finely dispersed structure.

A fragment of dispersed structure (fig. 5) is considered for researching the basic features of the current generation process in an object being under radiation. The cubic fragment of 0.003 $\mathrm{cm}$ size consists of binder (epoxy resin, density is about $1 \mathrm{~g} / \mathrm{cm}^{3}$ ) and one spherical inclusion (ammonium perchlorate, $\mathrm{NH}_{4} \mathrm{ClO}_{4}$, density is about $2 \mathrm{~g} / \mathrm{cm}^{3}$ ) of $0.002 \mathrm{~cm}$ diameter. The studied fragment is irradiated by photons of $20 \mathrm{KeV}$ energy in the direction of the $\mathrm{Z}$ axis.

\subsection{The main regularities of the generation of radiation-induced effects}

The distribution of fields of radiation-induced effects (heating, charge effects, electric currents) is determined mainly by the number of electrons born and their penetrating power. The first value is proportional to the macroscopic cross section of the interaction of photons with matter, and the second is proportional to the braking path of electrons. The dependence of these values on the energy of the radiation particles is shown in fig. 6,7 .

These figures show that in inclusion, the macroscopic cross-section of the interaction of photons significantly (up to two orders of magnitude) exceeds this value for the binder. Therefore, much more electrons are born in the inclusion than in the binder. The penetrating 
power (braking distance) of electrons, on the contrary, is noticeably greater in the binder.

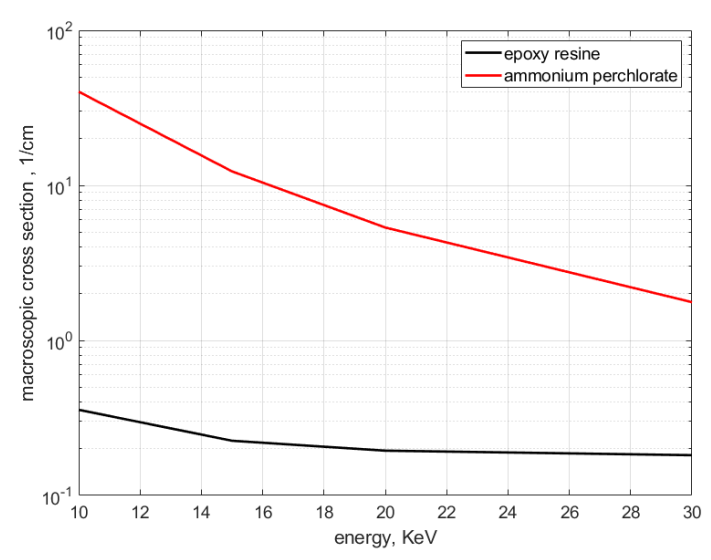

Fig. 6. Microscopic cross sections

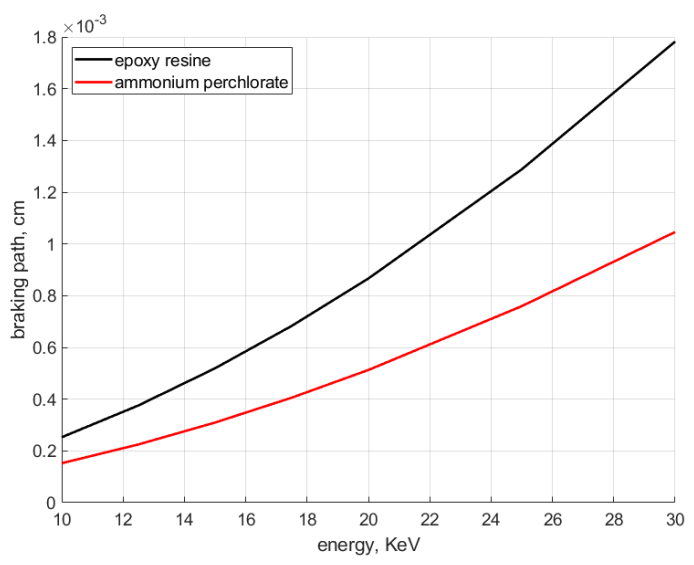

Fig. 7. Braking paths

The generation of electric currents in a substance being under gamma-or x-ray radiation is caused by the fluxes of photo and Compton electrons generated as a result of the photoelectron cascade processes in the material under study.

Current components for which there is no electronic equilibrium, that is, electron flows along the direction of this current component and in the opposite direction do not compensate for each other, will be different from zero at a given spatial point. The photon flux propagates along the $z$ axis in the computational experiment under consideration, so the transverse $(x, y)$ components of the current will obviously be negligible in a homogeneous medium.

The electron braking distance does not exceed 4 microns, and the number of collisions reaches tens and hundreds in the studied fragment of heterogeneous material. Therefore, at the periphery of the fragment (at 2-4 microns from the inclusion boundaries) in the binder, the absence of transverse current components should be expected.

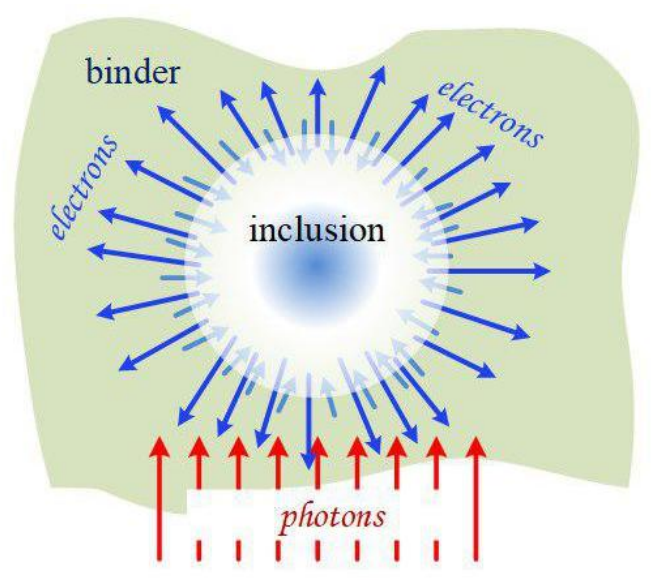

Fig. 8. Electron fluxes from and into the inclusion

Another situation is realized near the interface of two media with different physical properties (density, cross sections, braking paths) on spatial scales of the order of the electron path. The concentration of electrons born in the inclusion is much greater than in the binder. 
In addition, electrons emitted from the inclusion into the binder have a much greater penetrating power than electrons moving in the opposite direction (fig. 8).

Therefore, uncompensated electron fluxes arise near the boundaries of two media and the direction of the electron fluxes is from the inclusion into the binder. This direction is due to the predominance of electron emission from inclusion in the binder over emission in the opposite direction [23].

\subsection{Results of the modeling of radiation-induced electrical current}

The fig. 9-12 below show the spatial distributions of the amplitude of the transverse components $J_{x}$ and $J_{y}$ of the current in the irradiated fragment. These figures show the amplitudes of the electric current density in CGSE units per 1 photon $/ \mathrm{cm}^{2}$.

The fig. 9, 10 show graphs of the transverse components along straight lines $\{z=0.0015$ $\mathrm{cm}, \mathrm{y}=0.0015 \mathrm{~cm}\}$ and $\{\mathrm{z}=0.0015 \mathrm{~cm}, \mathrm{x}=0.0015 \mathrm{~cm}\}$ respectively. Dotted lines mark the boundaries of the inclusion.

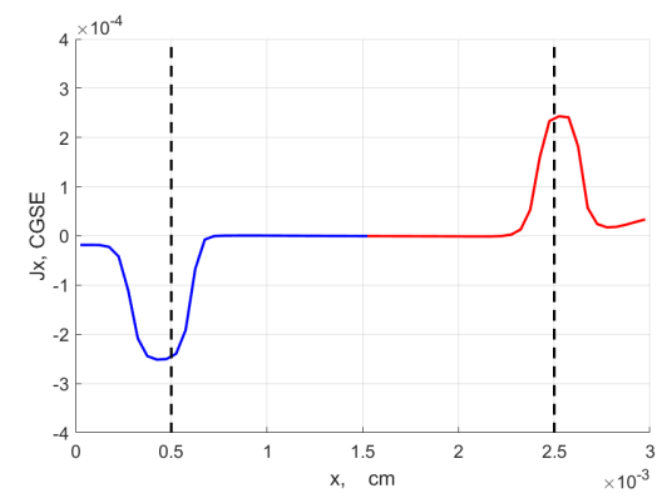

Fig. 9. The transverse component $J_{x}$

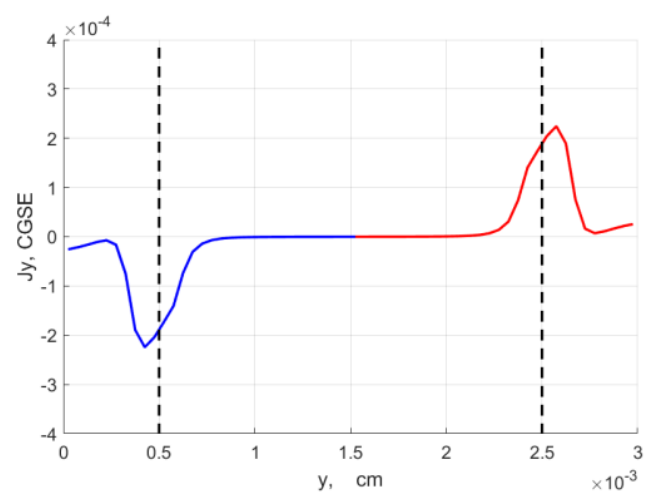

Fig. 10. The transverse component $J_{y}$

The transverse components of the current are negligible along the longitudinal axis $\{\mathrm{x}=0.0015 \mathrm{~cm}, \mathrm{y}=0.0015 \mathrm{~cm}\}$ passing through the "poles" of the inclusion.

Spatial distributions of the transverse components $J_{x}$ and $J_{y}$ in the plane $\mathrm{z}=0.0015 \mathrm{~cm}$ are shown in Fig. 11, 12 in the form of the surfaces.

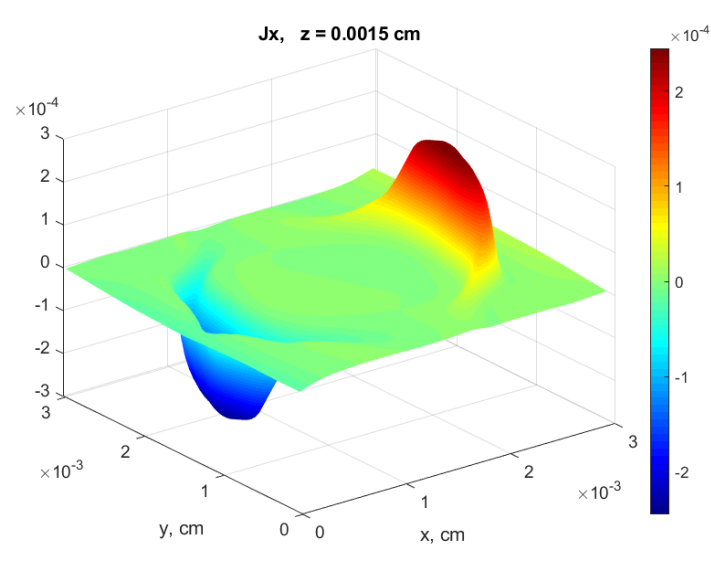

Fig. 11. 2D image of the component $J_{x}$

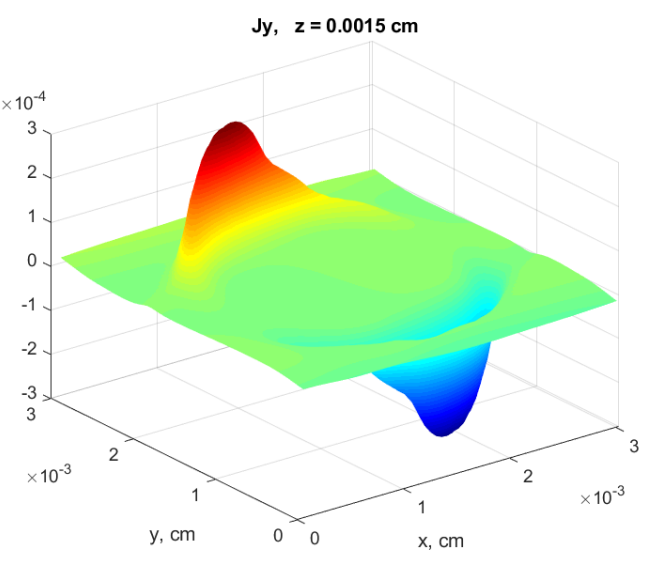

Fig. 12. 2D image of the component $J_{y}$ 
In General, these figures demonstrate the expected symmetry of the distribution of the transverse components of the current relative to the corresponding coordinate axes.

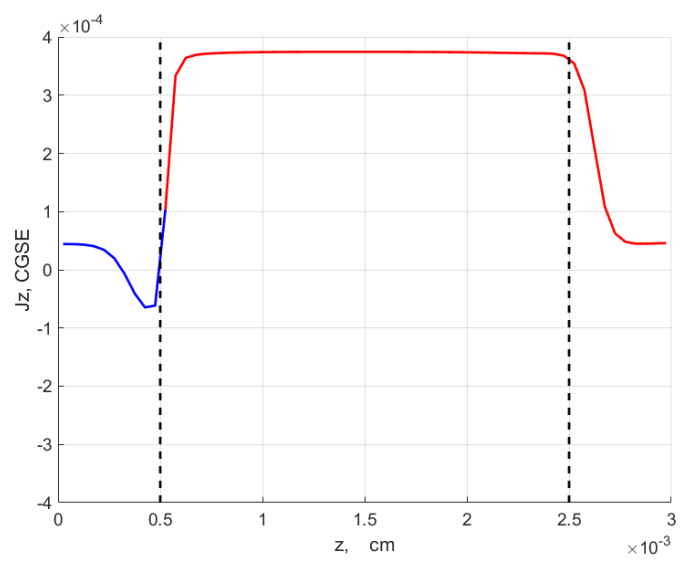

Fig. 13. $J_{z}$ component along the longitudinal axis $\{\mathrm{x}=0.0015 \mathrm{~cm}, \mathrm{y}=0.0015 \mathrm{~cm}\}$

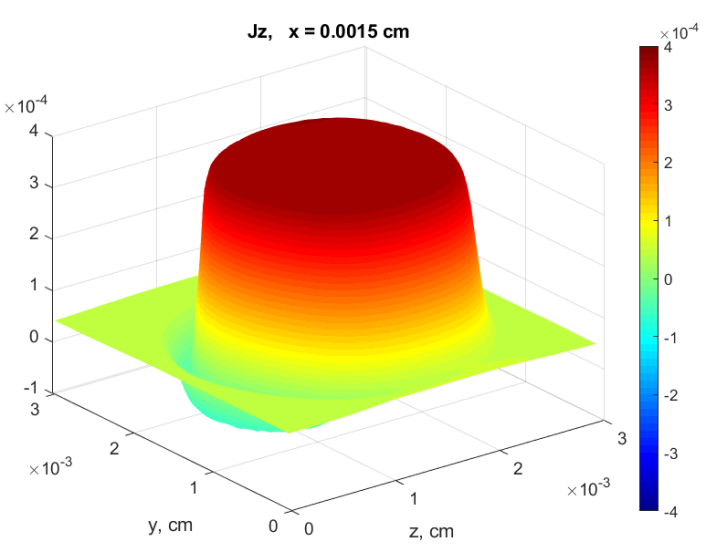

Fig. 14. 2D image of the component $J_{z}$

Fig. 13, 14 show the spatial distributions of the longitudinal (along the direction of the photon flow) component of the current $J_{z}$. The component $J_{z}$ in the plane $\mathrm{x}=0.0015 \mathrm{~cm}$ is shown in Fig. 14 in the form of the surface.

The component $J_{z}$ reaches the maximum value at the boundary of the two media because the electron emission from the inclusion into the binder is much more intense than in the opposite direction (fig. 8). The background longitudinal component of the current is generated at the periphery of the binder (at a distance from the boundary surfaces exceeding the braking path of the electron). Its value is significantly less than one of the inclusion-binder boundaries.

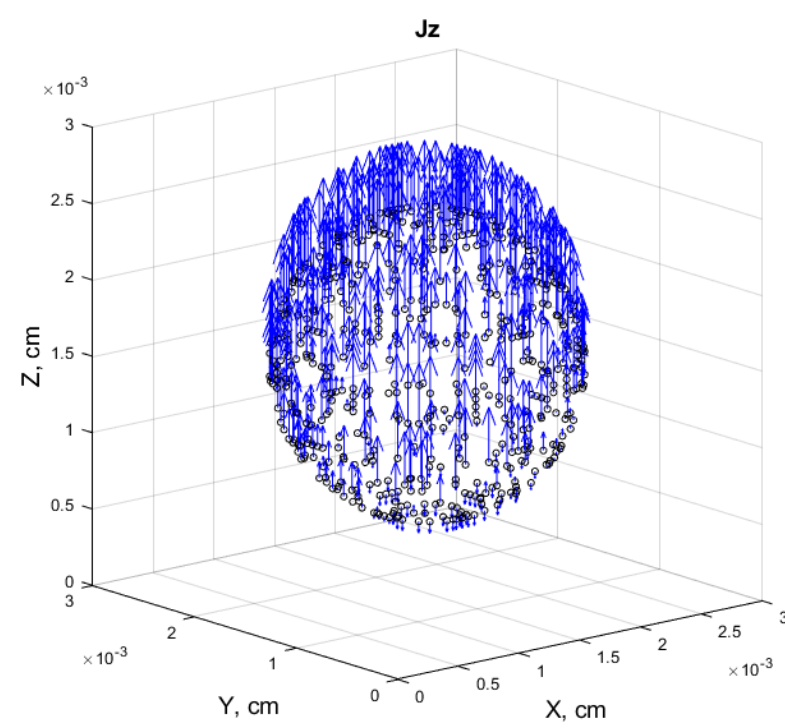

Fig. 15. $\mathbf{J}_{z}$

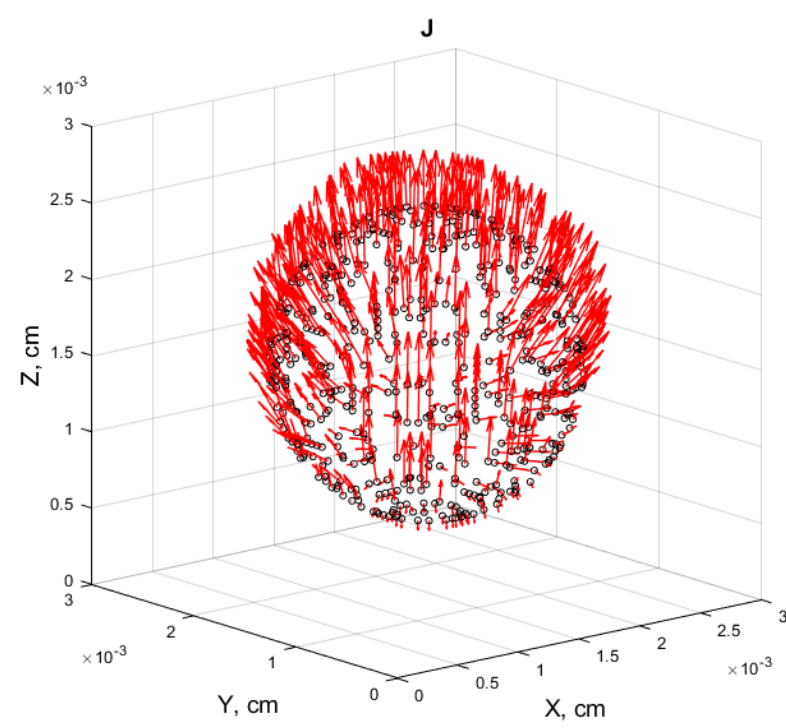

Fig. 16. $\mathbf{J}=\mathbf{J}_{\mathrm{x}}+\mathbf{J}_{\mathrm{y}}+\mathbf{J}_{\mathrm{z}}$ 
Vector field of the current in the binder near the boundary surface inclusion-binder is depicted in fig. $15\left(\mathbf{J}_{\mathrm{z}}\right)$ and fig. $16\left(\mathbf{J}=\mathbf{J}_{\mathrm{x}}+\mathbf{J}_{\mathrm{y}}+\mathbf{J}_{\mathrm{z}}\right)$.

These figures demonstrate the expected asymmetry of the electric current distribution with respect to the $\mathrm{z}=$ const plane passing through the center of the inclusion.

\section{CONCLUSION}

The technology of supercomputer simulation of radiation-induced electric currents in heterogeneous dispersed materials with direct consideration of their microstructure is developed. The main attention is paid to the creation of an algorithm for constructing a geometric model of a polydisperse medium, which is intended for implementation on heterogeneous computing clusters. The geometric model includes a detecting system for statistical evaluation of the desired physical quantities (electric current density).

The results of a computational experiment to calculate the current density in a fragment of finely dispersed material show that electric currents with a sharply inhomogeneous spatial structure are formed during irradiation of the object under study. Inhomogeneities occur near the boundary surfaces between the binder and inclusions. The generation of a current at the boundaries between two media is caused by the predominance of electron emission from the inclusion (a material with a large macroscopic cross-section of photons) in the binder (a material with a greater penetration of electrons) over the emission in the opposite direction (Fig. 8). Only the longitudinal component of the current (along the direction of the photon flow) is present in the binder at more than the length of the braking distance from the outer surface of the inclusion. Its value is much smaller than the amplitude of the current near the binder-inclusion boundary.

Acknowledgements: The work was financial supported by the Russian Scientific Foundation (project No. 17-71-30014).

\section{REFERENCES}

[1] A.V. Ostrik, A.I. Potapenko, "Geterogennye materialy dlia zashchity konstruktsii ot intensivnykh potokov energii”, Konstruktsii iz kompozitsionnykh materialov, 1, 48-53 (2001).

[2] Kondensirovannye energeticheskie sistemy, B.P. Zhukov (Ed.), Yanus-K (1999).

[3] A.M. Lipanov, A.V. Aliev, Proektirovanie RDTT, M.: Mashinostroenie (1995).

[4] A.R. Frederickson, L. Levy, C.L. Enloe, "Radiation-induced electrical discharges in complex structures", IEEE Trans. Electr. Insulation, 27 (6), 1166-1178 (1992).

[5] V.V. Gromov, Elektricheskii zariad v obluchennykh materialakh, M.: Energoizdat (1982).

[6] D.N. Sadovnichij, E.A. Golub, A.P. Tyutnev, A.V. Yushkov, "Raschet elektricheskikh polei v ploskikh geterogennykh strukturakh", Khimiia vysokikh energii, 5, 3-8 (1993).

[7] Dielektriki i radiatsiia, N.S. Kostyukov (Ed.), 3, M.: Nauka (2003).

[8] Yu.M. Milyoxin, D.N. Sadovnichij, S.A. Gusev, "Elektrizatsiia tverdotoplivnykh energeticheskikh ustanovok pri vozdeistvii ioniziruiushchego izlucheniia", Khimicheskaia fizika i mezoskopiia, 8 (1), 2006.

[9] A.V. Ostrik, "Prognozirovanie zashchitnykh svoistv pokrytii iz sferoplastikov pri vozdeistvii vysokointensivnykh potokov ioniziruiushchego izlucheniia", Deformatsiia $i$ razrushenie materialov, 2, 36-43 (2010).

[10] M.V. Alekseev and others, "O modelirovanii radiatsionno-indutsirovannykh zariadovykh effektov v melkodispersnykh materialakh zamknuto-iacheistoi struktury" Preprint IPM, 80 
(Moscow: KIAM), (2019). doi:10.20948/prepr-2019-80 URL: http://library.keldysh.ru/ preprint.asp?id=2019-80.

[11] V.I. Mazhukin, A.V. Shapranov, A.V. Mazhukin, "The structure of the electric double layer at the metal-vacuum interface", Mathematica Montisnigri, 44, 110-121 (2019).

[12] K.K. Inozemtseva, M.B. Markov, F.N. Voronin, "The electromagnetic and thermomechanical effects of electron beam on the solid barrier", Mathematica Montisnigri, 39, 79-100 (2017).

[13] M. E. Zhukovskiy, S. V. Podolyako, and R. V. Uskov, "Model of individual collisions for description of electron transport in matter," Mathematical Models and Computer Simulations, 4 (1), 101-109 (2012).

[14] M. E. Zhukovskiy and R. V. Uskov, "Hybrid Parallelization of the Algorithms of Radiation Cascade Transport Modelling", Mathematical Models and Computer Simulations, 7 (6), 601610 (2015).

[15] M. B. Markov, R. V. Uskov, M. E. Zhukovskiy, "Monte Carlo modelling of the photonelectron cascade in heterogeneous matter", Mathematica Montisnigri, 46, 49-60, (2019).

[16] F.H. Stillinger, B.D. Lubachevsky, "Crystalline-Amorphous Interface Packings for Disks and Spheres”, J. Stat. PhysicsB, 73 (3-4), 497-514 (1993).

[17] B.D. Lubachevsky, F.H. Stillinger, "Geometric properties of random disk packings", J. Stat. Physics, 60 (5-6), 561-583 (1990).

[18] V. A. Egorova, R. V. Uskov, F. N. Voronin and M. E. Zhukovskiy, "On the modeling of the thermomechanical fields in porous material being under radiation", Mathematica Montisnigri, 43, 58-69 (2018).

[19] M.D. Webb, I.L. Davis, "Random particle packing with large particle size variations using reduced-dimension algorithms", Powder Technology, 167 (1), 10-19 (2006).

[20] Y. Shi, Y. Zhang, "Simulation of random packing of spherical particles with different size distributions", Applied Physics A: Materials Science \& Processing, 92 (3), 621-626 (2008).

[21] A.A. Bolkisev, "O primenenii metoda sluchainogo poiska $\mathrm{k}$ zadache sluchainoy upakovki tverdykh chastic dlya modelirovaniya struktury smesevogo tverdogo topliva", Vesti Udmurtskogo Universiteta, Matem. Mekh. Kompyut. Nauki, 2, 106-113 (2012).

[22] M. Zhukovskiy, S. Podoliako, G.-R. Jaenisch, C. Bellon, U. Samadurau, "Monte-Carlo radiographic model with CAD-based geometry description". Insight, 48, (10), 618-623 (2006).

[23] M. Zhukovskiy, M. Markov, S. Podoliako and R. Uskov, "Hybrid parallelization of computing the electron fluxes produced by photon radiation", Mathematica Montisnigri, 31, 43-53 (2014).

Received November 10, 2019 